\title{
Algorithms for the ATLAS High-Level Trigger
}

S. Armstrong, J. T. Baines, C. P. Bee, M. Biglietti, A. Bogaerts, V. Boisvert, M. Bosman, S. Brandt, B. Caron, P. Casado, G. Cataldi, D. Cavalli, M. Cervetto, G. Comune, A. Corso-Radu, A. Di Mattia, M. Diaz Gomez, A. dos Anjos, J. Drohan, N. Ellis, M. Elsing, B. Epp, F. Etienne, S. Falciano, A. Farilla, S. George, V. Ghete, S. González, M. Grothe, A. Kaczmarska, K. Karr, A. Khomich, N. Konstantinidis, W. Krasny, W. Li, A. Lowe, L. Luminari, C. Meessen, A. G. Mello, G. Merino, P. Morettini, E. Moyse, A. Nairz, A. Negri, N. Nikitin, A. Nisati, C. Padilla, F. Parodi, V. Perez-Reale, J. L. Pinfold, P. Pinto, G. Polesello, Z. Qian, S. Resconi, S. Rosati, D. A. Scannicchio, C. Schiavi, T. Schoerner-Sadenius, E. Segura, J. M. Seixas, T. Shears, S. Sivoklokov, M. Smizanska, R. Soluk, C. Stanescu, S. Tapprogge, F. Touchard, V. Vercesi, A. Watson, T. Wengler, P. Werner, S. Wheeler, F. J. Wickens, W. Wiedenmann, M. Wielers, and H. Zobernig

\begin{abstract}
Following rigorous software design and analysis methods, an object-based architecture has been developed to derive the second- and third-level trigger decisions for the future ATLAS detector at the LHC. The functional components within this system responsible for generating elements of the trigger decisions are algorithms running within the software architecture. Relevant aspects of the architecture are reviewed along with concrete examples of specific algorithms and their performance in "vertical" slices of various physics selection strategies.
\end{abstract}

Index Terms-Algorithms, online reconstruction, trigger.

Note: This paper was presented by Stephen Armstrong on behalf of the ATLAS High Level Trigger Group [1].

\section{INTRODUCTION}

\section{A. Overview of the Large Hadron Collider (LHC)}

$\mathbf{T}$ HE future LHC project at CERN, Geneva, Switzerland, will be a colliding proton synchrotron with a center-of-mass energy of $14 \mathrm{TeV}$. It is anticipated to deliver an initial luminosity of $2 \times 10^{33} \mathrm{~cm}^{-2} \mathrm{~s}^{-1}$ to two large general purpose detector experiments (ATLAS and CMS) and scale up to the full design luminosity of $10^{34} \mathrm{~cm}^{-2} \mathrm{~s}^{-1}$. The high final state particle multiplicities from the proton-proton collisions necessitate highly granular and large scale detector systems producing 1 to 2 MByte event sizes from $10^{8}$ electronic channels. The bunch crossing rate of $40 \mathrm{MHz}$ implies fast trigger and data acquisition (DAQ). When these factors are considered along with the high radiation environment in which the detectors and their electronics must function for the decade-long lifetime of the experiment, the LHC program places unprecedented and extreme demands on detectors and trigger/DAQ systems.

\section{B. Atlas Detector}

The ATLAS detector [2] consists of several highly granular and hermetic concentric subdetector systems oriented coaxially

Manuscript received June 5, 2003; revised February 18, 2004.

The authors are with ATLAS High-Level Trigger Group, PH Department CERN, CH-1211 Geneva 23, Switzerland (e-mail: Steven.Armstrong@ cern.ch).

Digital Object Identifier 10.1109/TNS.2004.828620 with respect to the LHC beamline and centered around the nominal proton-proton collision point. This subsection briefly summarizes details of the detector relevant to the discussion below. Throughout this document, the LHC beamline is referred to as the $z$ axis; kinematic variables such as transverse momentum $p_{\mathrm{T}}(\mathrm{GeV} / c)$ are defined from this axis as is the polar angle $\theta$ from which pseudorapidity $\eta=-\ln (\tan (\theta / 2))$ is derived.

Three subdetector systems at the innermost radii constitute the Inner Detector (InDet) tracking system: the Pixel detector, the Semiconductor Tracker (SCT), and the Transition Radiation Tracker (TRT). These detectors are immersed in a $2 \mathrm{~T}$ axial magnetic field generated by a superconducting solenoid magnet outside the TRT. By reconstructing hits left from charged particles traversing the fiducial tracking volume, high efficiency tracking with good impact parameter resolution can be achieved up to an absolute pseudorapidity of 2.5. The InDet provides a reconstruction efficiency greater than $90 \%$ over a broad $p_{\mathrm{T}}$ spectrum for isolated tracks with resolutions of $\sigma\left(1 / p_{\mathrm{T}}\right)=0.36 \oplus 13 /\left(p_{\mathrm{T}} \sqrt{\sin \theta}\right)\left(\mathrm{TeV}^{-1}\right)$ and $\sigma\left(d_{0}\right)=11 \oplus 73 /\left(p_{\mathrm{T}} \sqrt{\sin \theta}\right)(\mu \mathrm{m})$.

A liquid Argon calorimeter (LAr) with an accordion-shaped electrode design is situated outside the solenoid magnet and provides electromagnetic calorimetery within $|\eta|<3.2$ with a resolution of $\sigma_{\mathrm{E}} / E=10 \% / \sqrt{E} \oplus 0.7 \% \oplus 0.27 / E$ and a position resolution of $\sigma_{\theta}=40 \mathrm{mrad} / \sqrt{E}$. The LAr is segmented into three longitudinal sections referred to as samplings. The first of these samplings is equipped with narrow strips of $\sim 4 \mathrm{~mm}$ in the $\eta$ direction and aids in particle identification. Outside the LAr is a hadron calorimeter with a novel radial scintillating tile geometry (Tile) providing a resolution of $\sigma_{\mathrm{E}} / E=50 \% / \sqrt{E} \oplus$ $3 \%$. LAr technology is also used for hadron calorimetery in the endcap regions as well as special forward calorimeters extending the coverage to $|\eta|=4.9$.

Outside the calorimetry system is an air-core Barrel Toroid (BT) and Endcap Toroid (ECT) magnet system interleaved by, and surrounded with, several types of tracking chambers comprising the Muon Spectrometer. The peak field intensities are 3.9 and $4.1 \mathrm{~T}$ in the BT and ECT respectively. There are two types of fast response chambers contributing to the Level-1 Trigger decision:Thin Gap Chambers (TGC) and Resistive Plate Chambers (RPC). There are also two types of precision tracking chambers: Monitored Drift Tubes (MDT) and Cathode 
Strip Chambers (CSC). The muon spectrometer provides a standalone momentum resolution of roughly $2 \%$, allowing it to identify muons up to $1 \mathrm{TeV} / c$.

The physical size of the detector is defined by the outermost chambers of the muon spectrometer: $46 \mathrm{~m}$ long, with a diameter of $22 \mathrm{~m}$. The overall mass of the detector is roughly $7 \times 10^{6} \mathrm{~kg}$.

\section{Trigger System}

The ATLAS trigger system must accept the high $40-\mathrm{MHz}$ bunch crossing frequency and reduce it to a manageable rate of roughly $200 \mathrm{~Hz}$. It is comprised of a three-level system. The first-level hardware-based trigger (Level-1) quickly analyzes data from the calorimeter and muon spectrometer systems to derive an accept or reject decision within $2 \mu$ s. Events are passed on at a rate of $75 \mathrm{kHz}$ to a second-level software-based trigger (Level-2), which must derive a decision within an average latency of $10 \mathrm{~ms}$. Level-2-accepted events are passed on at a rate of roughly $3 \mathrm{kHz}$ to the third-level software-based Event Filter (EF), which has a more generous latency of roughly $1 \mathrm{~s}$ to pass the event on to offline mass storage at a rate of roughly $200 \mathrm{~Hz}$. It is axiomatic that only events surviving this three-stage triggering system can be part of subsequent physics analysis. Together, the Level-2 and EF are referred to as the High-Level Trigger (HLT) [3].

To achieve the required rejection power while retaining sensitivity to signal events within the broad ATLAS physics program, components of physics analysis traditionally deferred to offline environments must be embedded within the trigger system. Hence, the first stages of ATLAS physics analysis reside and must be understood within the online trigger system. Table I provides examples of physics objects, the corresponding trigger element (TE) nomenclature for them, and the area of physics analysis reliant upon them. The TEs denote and characterize abstract physics objects with a succinct label (e.g., "e20i" for an isolated $20 \mathrm{GeV}$ electron).

\section{Architecture of the High-LeVel TrigGer Selection SOFTWARE (HLTSS)}

Fig. 1 provides a disaggregated view of the key components of the HLTSSW. The HLTSSW runs on dedicated hardware consisting of farms of PCs interfaced to the ATLAS Data Collection Systems. For Level-2, the components of the HLTSSW must behave in a multithreaded-safe manner.

\section{A. HLT Algorithm Strategy}

Algorithms reconstruct objects and extract features from event data; these features are used to derive the trigger decision. At Level-2, highly specialized algorithms use a restricted portion of event data usually defined in terms of Regions-of-Interest (RoI) derived from the Level-1 decision. Modified algorithms from the offline software are used as EF algorithms and have full access to event data. In both cases, algorithms must be capable of being seeded from results derived at a previous stage of the trigger chain. Furthermore, they may be called multiple times per event (e.g., in the case of multiple RoIs found at Level-1 in a single event). Hence, they do not operate in a general purpose mode as in the offline software, but
TABLE I

EXAMPLES OF PHYSICS OBJECTS, THE CORRESPONDING TRIGGER ELEMENT NOMENClature, AND AREAS of Physics COVERAge. ThE TRIGgER ElEMENT NOMENCLATURE IS STRUCTURED AS $(N) X p_{\text {T }}$ (i) WHERE $N$ REFERRS TO THE Multiplicity of ReQuREd Objects Described, $X$ Denotes Final State PHYSICS OBJECTS ("e" FOR EleCtrons, " $\mu$ " FOR MUONS, " $\tau$ ” FOR TAU LEPTONS, " $\gamma$ ” FOR PHOTONS, "j" FOR JeTS, "xE" FOR MISSING ENERGY), $p_{\mathrm{T}}$ DENOTES A MINIMUM $p_{\text {T }}$ CRITERION, AND "i” FOR ANY ISOLATION CRITERIA

\begin{tabular}{c|c|c}
\hline Object & Nomenclature & Coverage \\
\hline electrons & e25i, 2e15i & $\begin{array}{c}\text { Higgs (SM, MSSM), new gauge } \\
\text { bosons, extra dimensions, } \\
\text { SUSY, W, top }\end{array}$ \\
\hline photons & $\gamma 60 \mathrm{i}, 2 \gamma 20 \mathrm{i}$ & $\begin{array}{c}\text { Higgs (SM, MSSM), extra } \\
\text { dimensions, SUSY }\end{array}$ \\
\hline muons & $\mu 20 \mathrm{i}, 2 \mu 10$ & $\begin{array}{c}\text { Higgs (SM, MSSM), new gauge } \\
\text { bosons, extra dimensions, } \\
\text { SUSY, W, top }\end{array}$ \\
\hline jets & $\mathrm{j} 360,3 \mathrm{j} 150,4 \mathrm{j} 100$ & SUSY, compositeness, resonances \\
\hline jets $+\mathbb{E}_{\mathrm{T}}$ & $\mathrm{j} 60+\mathrm{xE60}$ & SUSY, leptoquarks \\
\hline tau $+\mathbb{E}_{\mathrm{T}}$ & $\tau 30+\mathrm{xE40}$ & extended Higgs models, SUSY \\
\hline
\end{tabular}

rather must work in a Bayesian-like environment by validating only specific hypotheses given a certain seed.

\section{B. Event Data Model}

To facilitate the importation of algorithms from the offline software, as well as to permit a configurable continuum of selection in the HLT by means of interchangeable Level- 2 and EF algorithms, a common Event Data Model (EDM) is essential. The EDM is the common language within and between algorithms defining the objectified representation of event data referred to as event data objects (EDOs). The establishment of a common EDM within the ATLAS offline and online software environments is an ongoing effort [4].

In the discussion below, relevant EDOs are as follows.

- Clusters of hits within the Pixel(SCT) detector are two(one)-dimensional groups of neighboring readout channels in a Detector Element.

- Clusters are converted into three-dimensional SpacePoints which are used to determine a track defining the trajectory of a charged particle.

- Straw tubes in the TRT detector can measure the distance of ionization from its central wire by measuring the drift time. These measurements are referred to as DriftTimeHits. If the drift time is not available, a default spatial resolution given by the straw diameter divided by $\sqrt{12}$ is used instead, and it is referred to as a StrawHit. The TRT electronics has two independent thresholds for signals; signals passing a higher threshold are likely to be due to transition radiation and are referred to as TRHits.

- A calorimeter Cell corresponds to the LAr readout granularity; this corresponds to a $\Delta \eta \times \Delta \phi$ size of $0.003 \times$ $0.1,0.025 \times 0.025$, and $0.05 \times 0.025$ in Samplings 1, 2, and 3 respectively.

- A calorimeter CaloCluster is a grouping of Cells in a $\Delta \eta \times \Delta \phi$ window. The determination of the window size for a CaloCluster is determined by studying contributions to the energy measurement from low-energy 


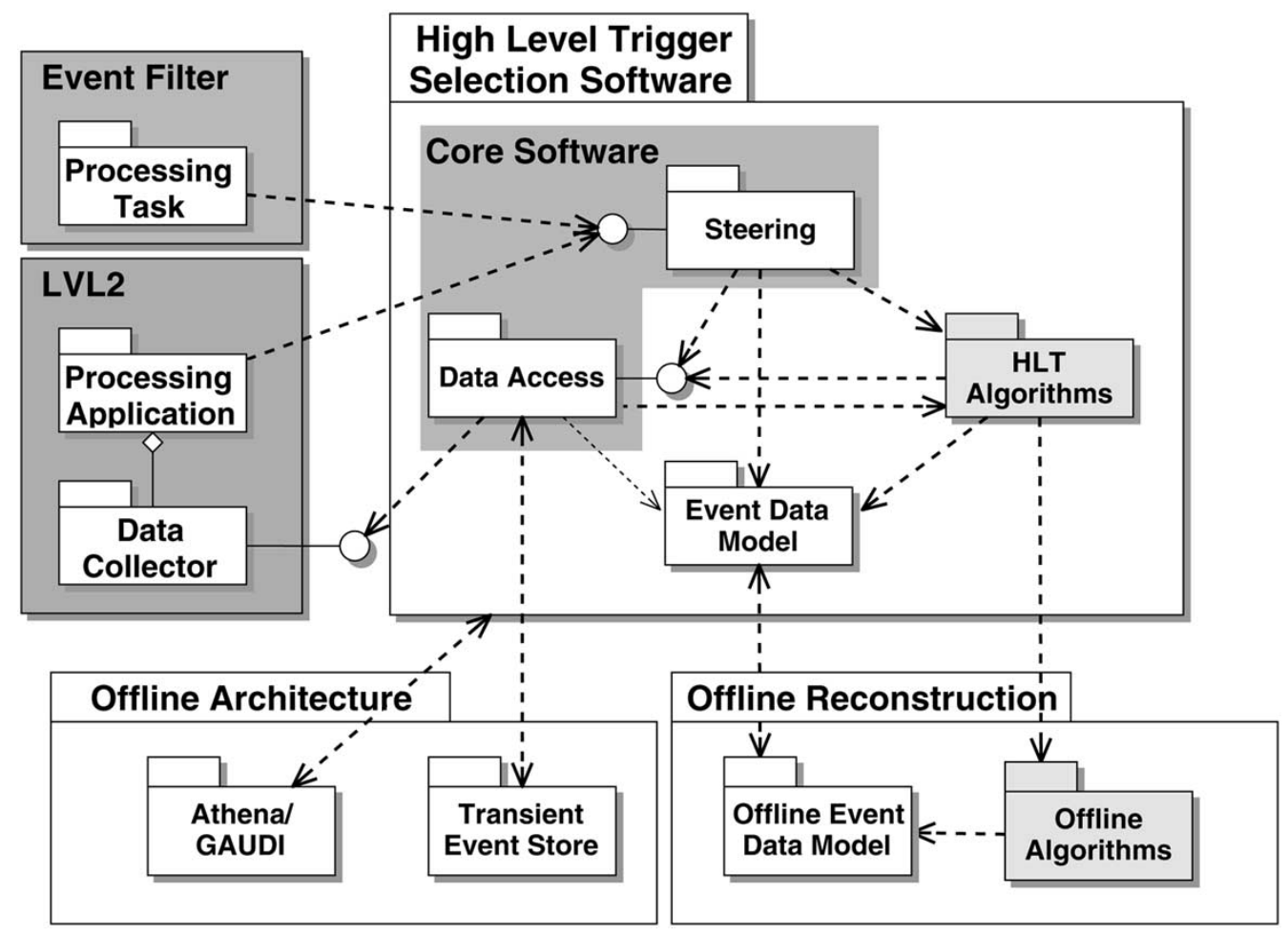

Fig. 1. A package diagram illustrating the key components of the HLTSSW.

tails, fluctuations in the energy leakage outside the CaloCluster, electronic noise, and pile-up. A $3 \times 7 \mathrm{Cell}$ window size in the second LAr Sampling is optimal for electrons and converted photons with energies less than $100 \mathrm{GeV}$ [16].

\section{Importation of the Offline Software Framework}

In addition to algorithms, the HLTSSW imports and uses key components of the ATLAS object-oriented C++ Offline Software framework, referred to as Athena [5]. Within this framework, there is a separation of algorithmic objects from EDOs. The EDM specifies the nature and content of EDOs which are passed between algorithms and software packages via a transient event store (TES). A corollary to this approach is that, contrary to canonical object-oriented programming, data object classes contain minimal algorithmic content (e.g., algorithms for finding, following, or fitting tracks are separated from methods in the class definition of the Track itself).

\section{Restricted Data Access}

At Level-2, algorithms actively request portions of event data from the Data Collection System. The relevant data are defined by RoIs based on information from the decision from Level-1 or a previous result in Level- 2 processing. For each RoI, the total data volume with respect to the whole detector is roughly a few percent. The number of RoIs is dependent upon the RoI-type (e.g., electromagnetic, jets, etc.) and luminosity conditions, varying between 1 and 6 [6]. Hence, this restricted data access strategy represents a significant reduction in the required HLT processing and networking resources.

For a given RoI, typically defined by an extent in $\eta$ and $\phi$ within the physical detector volume, a Region Selector [7] translates the physical volume into a set of offline identifiers [8]. These identifiers are translated at a subsequent stage (see Section II-E) into online identifiers which may then be used to request the data themselves.

It may seem counterintuitive to use such a scheme (i.e., conversion into a geometrical region which requires translation into Offline identifiers which then require translation into Online identifiers). There are, however, a variety of motivations for the Region Selector. The prime motivation is to gain access in a uniform and rapid way to event data from subdetectors which do not participate in the Level-1 trigger decision (e.g., event data from InDet tracking information given a Level-1 trigger based on an energy deposit in the Calorimeters). An additional motivation includes allowing for possible secondary RoIs as needed by an algorithm which may lie outside the primary RoI defined by Level-1.

\section{E. Paradigm for Realistic Raw Data Access}

Raw data from the ATLAS detector will be delivered in terms of a ByteStream of data consisting of hierarchically arranged fragments formatted in a subdetector-dependent way. This ByteStream of data must be converted into EDOs which can then be used by algorithms. Modeling this flow and conversion of ByteStream data in a realistic way is vital to an accurate modeling of the HLT performance and subsequent estimation of required network and computing resources.

The HLTSSW adopts a scheme whereby the interaction of HLT algorithms with the Data Collection System is hidden behind a call to the TES. Fig. 2 illustrates this scheme. An algorithm requests data within a certain region by first feeding the parameters of the region to the Region Selector. The Region Selector returns a set of Offline Identifiers which the algorithms 


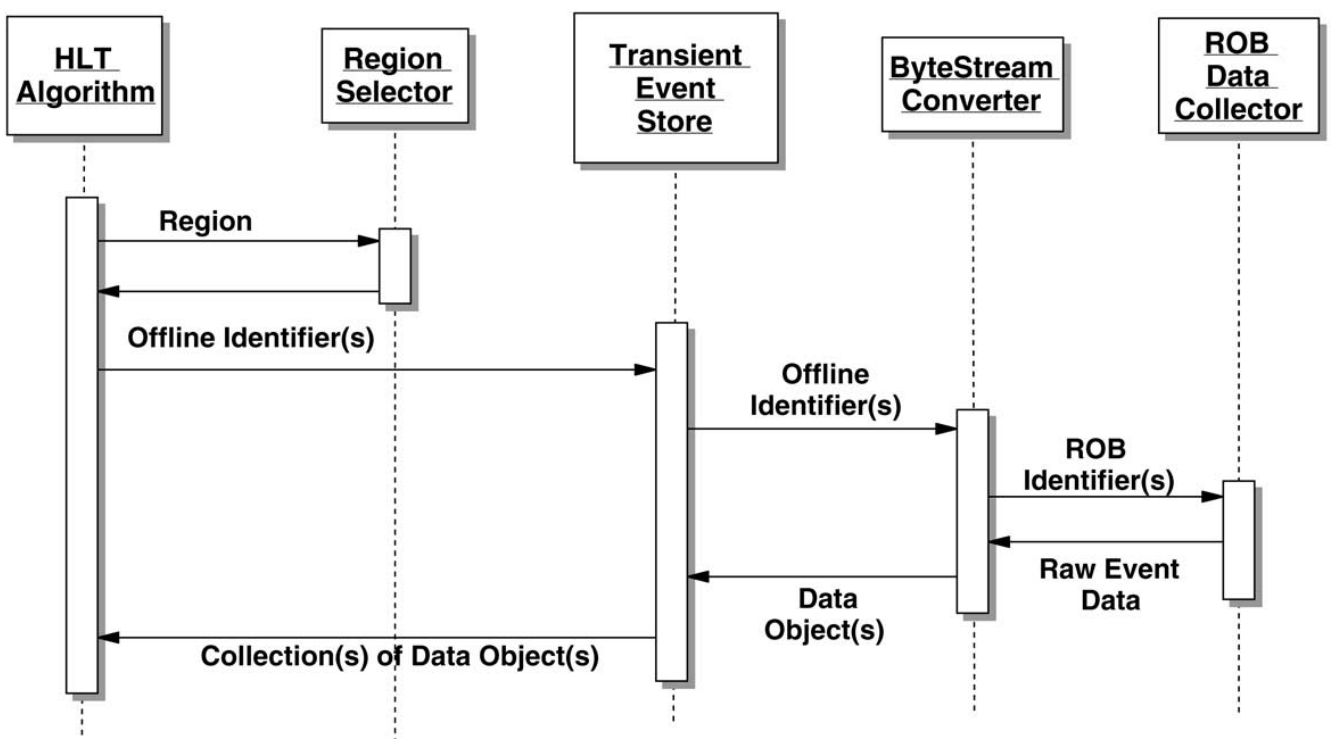

Fig. 2. A simplified schematic diagram of the sequence by which HLT algorithms request and receive event data.

then uses to request collections of relevant EDOs from the TES. If the TES does not contain the EDOs, it requests these data from a RawDataConverter. The Offline Identifiers are translated into Online or readout buffer (ROB) Identifiers which are used to request the data from the Data Collection System. Table II gives the current granularity of the ATLAS detector in terms of the number of Offline and ROB Identifiers used for each subdetector system. The raw data returned from the Data Collection System are in ByteStream format and are converted into EDOs and stored in the TES in collections tagged with Offline Identifiers. The TES then returns the collections of EDOs the algorithm originally requested.

\section{F. Guidance and Seeding of Algorithms}

The component of the HLTSSW which guides and steers algorithms is referred to as the Steering [9]. The Steering allows a fast and early rejection of uninteresting events in a flexible and configurable manner; the Steering also permits the pre-scale/forced-accept of some events. This is realized in a way that permits full control of the algorithms executing within the HLT processing flow by the modification of Extensible Markup Language (XML) configuration files.

The Step Controller (SC) of the Steering software replaces the application manager responsible within the offline software environment for iterating over events. Hence, the SC has the responsibility of calling algorithms. Two XML files encode $\mathrm{Se}$ quences and Signatures that in turn instruct the Steering when and how to run an algorithm and if a physics signature is fulfilled. Signatures and Sequences are built upon TEs. This decouples the Steering from the details of the EDM. A Navigation scheme relates TEs to each other and to underlying concrete EDOs. Hence, a TE can be thought of as the entry point for an algorithm into an event.

The HLT processing flow is disaggregated into Steps. Input TEs provide seeds to algorithms executing in each Step. The decision to go further in the process is taken at every new Step by
TABLE II

THE CURRENT GRANULARITY OF THE ATLAS DETECTOR FOR EACH SUB-DETECTOR SYSTEM IN TERMS OF THE DEFINITION OF THE OFFLINE UNIT OF GRANULARITY (UNIT), NUMBER OF OFFLINE IDENTIFIERS (OFF. ID.), AND ONLINE ROB IDENTIFIERS ROB ID

\begin{tabular}{c||c|c|c}
\hline & Unit & Off. Id. & ROB Id. \\
\hline Pixels & module & 1744 & 81 \\
SCT & side of module & 8176 & 256 \\
TRT & straw layer & 19008 & 256 \\
LAr & Trigger Tower & 7168 & 768 \\
Tile & module & 256 & 32 \\
MDT & chamber & 1168 & 192 \\
CSC & chamber & 32 & 32 \\
RPC & chamber & 574 & 32 \\
TGC & chamber & 1584 & 32 \\
\hline
\end{tabular}

the comparison between active TEs in the TES and the corresponding configuration Signature. An event is accepted if all its constituent Sequences have been executed and at least one of the corresponding configuration Signatures has been satisfied.

\section{HLT ALGORITHMS}

This section describes algorithms intended to be executed within the HLTSSW. An emphasis is placed on the more optimized and specialized algorithms developed for Level- 2 which must cope with more restrictive data access and latency.

In the case of Level-2 track reconstruction involving the precision Pixel and SCT subdetectors, two parallel algorithms have been developed: IDSCAN (described in Section III-A) and SiTrack (described in Section III-B). Likewise, in the case of Level-2 track reconstruction in the TRT subdetector, two parallel algorithms have also been developed: TRTxKalman (described in Section III-C) and TRT-LUT (described in Section III-D). This dual algorithm approach has proved beneficial in terms of allowing cross-checks, rapid development, and redundancy. 


\section{A. Level-2 Track Reconstruction: IDSCAN}

Taking as input SpacePoints found in the Pixel and SCT subdetectors, the Level-2 tracking algorithm IDSCAN [3] consists of a series of subalgorithms: ZFinder, HitFilter, Groupcleaner, and TrackFitter.

- The ZFinder finds the z-position of the primary interaction vertex, which has a one standard deviation spreadqq of $\pm 5.6 \mathrm{~cm}$ due to the LHC bunch size; this component of the algorithm is described in detail in [10]. All SpacePoints are placed into narrow $\phi$-bins and pairs of SpacePoints in each bin are extrapolated back to the beam-axis, storing the $z$ of intersection in a histogram. The $z$-position is then the histogram region with the most entries.

- The Hitfilter finds groups of spacepoints compatible with Tracks from the $z$ position found by ZFinder; it is described in detail in [11]. It puts all SpacePoints into a histogram binned in $\phi$ and $\eta$. It then finds clusters of hits within this histogram. It creates a group if such a cluster has SpacePoints in more than a given number of layers.

- The group found by HitFilter is used by GroupCleaner which splits groups into Tracks and removes noise from groups. Each triplet of hits forms a potential track for which $p_{\mathrm{T}}, \phi_{0}$, and $d_{0}$ are calculated. It forms groups from these triplets with similar parameters, applying certain quality cuts. It accepts a track candidate if a group contains enough hits.

- Finally, the TrackFitter verifies track candidates and determines the track parameters using a standard Kalmanfilter-type fitting algorithm. It returns a list of SpacePoints on the Track, the Track parameters, and an error matrix.

\section{B. Level-2 Track Reconstruction: SiTrack}

SiTrack [12] takes Pixel and SCT SpacePoints as input and provides fitted reconstructed Tracks, each containing links to the SpacePoints used to build it. SiTrack is implemented as a single main algorithm that executes a user defined list of subalgorithms described below.

- STSpacePointSorting collects pointers to SpacePoints coming from the Pixel and SCT detectors and sorts them by module address, storing the result in a Standard Template Library (STL) map. This processing step is performed in order to speed-up data access for the other reconstruction subalgorithms.

- In the case of a trigger with a high- $p_{\mathrm{T}}$ muon, the STMuonVertex primary vertex identification algorithm is used and is most suitable for low luminosity events. It is based on track reconstruction performed inside a Level-1 muon RoI: the most impulsive track is assumed to be the muon candidate, and its $z$ impact parameter is taken as the $z$ position of primary vertex position.

- STTrackSeeding, using the sorted SpacePoint map and a lookup table (LUT) linking each module within the innermost Pixel layer (B-layer) to the ones belonging to other logical layers, builds track seeds formed by two
SpacePoints and fits them with a straight line; one or more logical layers can be linked to the B-layer to improve efficiency. If the primary vertex has already been reconstructed by STMuonVertex, a fraction of fake track seeds can be rejected during their formation with a cut on their $z$ distance from the primary vertex. Otherwise, if no vertex information is available, a histogram whose resolution depends on the number of seeds found is filled with the $z$ impact parameter of each seed; its maximum is then taken as $z$ position for the primary vertex. This vertexing algorithm is most suitable for high luminosity events containing many high $p_{\mathrm{T}}$ tracks (e.g., b-tagging). Independent cuts on $r-\phi$ and $z$ impact parameters are eventually applied to the reconstructed seeds to further reduce the fake fraction.

- STThreePointFit extends track seeds with a third SpacePoint; it uses a map associating to each seed a set of module roads. Here, a road is a list of modules ordered according to the radius at which they are placed starting from the innermost one. A track may have hits in the Pixel or SCT detectors. A subset of modules is extracted from each road according to a user-defined parameter related to their depth inside it (e.g., the user can decide to use modules at the beginning or in the middle of each road, etc.). SpacePoints from the selected modules are then used to extend the seed and candidate tracks are fitted with a circle; ambiguities (e.g., tracks sharing at least one SpacePoint) can be solved on the basis of the track quality, leading to an independent set of tracks that can be used for trigger selection or as a seed for further extrapolation.

\section{Level-2 Track Reconstruction: TRTxKalman}

TRTxKalman utilizes StrawHits and DriftTimeHits from the TRT to reconstruct Tracks. The core of the algorithm is based upon a Hough-transform and borrows a set of utilities from the offline reconstruction package xKalman [13].

Pattern recognition is performed in a two-dimensional $\phi-$ $r(z)$ space for the TRT barrel (endcap). A lower $p_{\mathrm{T}}$ bound of $0.5 \mathrm{GeV} / c$ is applied to permit straight-line approximations to be used for particle trajectories. The trajectory of a particle with an initial azimuthal angle $\phi_{0}$, longitudinal position $z_{0}$, electriccharge $Q$, in a uniform magnetic field $H$ (in Tesla) oriented parallel to the beam-axis $z$, can be approximated as $\phi=\phi_{0}+$ $r c_{\mathrm{T}} / 2$ in the $\phi-r$ projection, and $\phi=\phi_{0}^{\prime}+\left(z c_{\mathrm{z}}\right) / 2$ in the $\phi-z$ projection. Here $c_{\mathrm{T}}=0.003 q H / p_{\mathrm{T}}$ and $c_{\mathrm{z}}=c_{\mathrm{T}} \tan \theta$ are the transverse and longitudinal curvatures, and $\phi_{0}^{\prime}=\phi_{0}-$ $\left(z_{0} c_{\mathrm{T}} \tan \theta\right) / 2$. However, the axial magnetic field in the InDet deviates significantly from uniformity for $\|z\|>2 \mathrm{~m}$; hence, a fourth-order Runge-Kutta method is used to calculate particle trajectories in these regions.

The pattern recognition occurs in the following steps.

- Using a Hough transformation, a histogram binned in terms of $\phi_{0}$ and $c_{\mathrm{T}}$ (or $c_{\mathrm{Z}}$ ) is filled with positions of StrawHits within an RoI. In this parameter space, each StrawHit can occupy many positions. Track candidates are identified from peaks in the histogram. 
- Narrow roads are defined using the Track candidates, and local pattern recognition is performed using DriftTimeHits and StrawHits within this region. Utilizing a recursive algorithm considering sets of possible track rotations around a given boundary segment of straw endpoints, final tracks and their parameters are extracted. This procedure is described in detail in [13].

- Tracks must satisfy quality criteria on the number of unique hits and ratio of hits to number of straws crossed. Each track contains a link to the number of TRHits which may also be used for electron identification at Level-2.

\section{Level-2 Track Reconstruction: TRT-LUT}

TRT-LUT [14] is an algorithm for track reconstruction in the TRT. The algorithm takes as input StrawHits within an RoI and provides Track candidates and their parameters as well as the StrawHits used for each Track; work is underway to incorporate DriftTimeHits into this algorithm.

The algorithmic processing consists of initial track finding, local maximum finding, track splitting, and track fitting and final selection.

- Initial Track Finding: every StrawHit in a three-dimensional representation of the TRT is allowed to belong to a number of possible predefined tracks characterized by different parameters. All such tracks are stored in a lookup table (LUT). Every StrawHit increases the probability that a track is a genuine candidate by one unit.

- Local Maximum Finding: a two-dimensional histogram is filled with bins in $\phi$ and $Q / p_{\mathrm{T}}$ for the track parameters at the primary vertex ( $Q$ denotes the electric-charge sign). A histogram for a single track consists of a "bow-tie"shaped region of bins with entries at a peak in the center of the region. The bin at the peak of the histogram will, in an ideal case, contain all the StrawHits from the Track. The roads corresponding to other filled bins share StrawHits with the peak bin, and thus contain subsets of the StrawHits from the track. A histogram for a more complex event would consist of a superposition of entries from individual tracks. Hence, bins containing a complete set of StrawHits from each track can be identified as local maxima in the histogram.

- Track Splitting: the pattern of StrawHits associated to a track candidate is analyzed. By rejecting fake candidates composed of StrawHits from several low- $p_{\mathrm{T}}$ tracks, this step results in an overall reduction in the number of track candidates by roughly a factor of two. For roads containing a good track candidate, it identifies and rejects any additional StrawHits from one or more other tracks. This results in a candidate that consists of a subset of the StrawHits within a road.

- Track Fitting and Final Selection: to improve the measurement of $\phi$ and $p_{\mathrm{T}}$ over that of a simple straight-line fit, a fit is performed in the $\phi-r(z)$ plane for the barrel (end-caps) using a third-order polynomial. This is given as $\phi-\left(\phi-\phi_{0}\right)^{3}=\phi_{0}+Q C r$ where $Q$ if the electric-charge and $C$ is the curvature. The track is assumed to come from the nominal origin. After the fit, a reconstructed $p_{\mathrm{T}}$ threshold of $0.5 \mathrm{GeV} / c$ is applied.

\section{E. Level-2 Calorimetry: T2CALO}

T2Calo [15] is a clustering algorithm for electromagnetic (EM) showers. It is seeded by Level-1 electromagnetic (EM) trigger RoI positions. This algorithm takes calibrated calorimeter Cells as input and provides discriminating variables to separate isolated $\mathrm{EM}$ objects from jets using $E_{\mathrm{T}}$ measurements and shower-shape quantities.

The first step of T2Calo is the refinement of the Level-1 position with the use of the position of the highest energy Cell in the second sampling of the EM calorimeter. The position $\left(\eta_{1}, \phi_{1}\right)$ of the highest energy Cell is later refined by calculating the energy weighted Calocluster position $\left(\eta_{c}, \phi_{c}\right)$ in a window of $3 \times 7$ in the second sampling.

After the first step, the algorithm proceeds to calculate each of the following variables and permits at each stage the application of selection criteria.

- In the second sampling, the quantity $R_{\eta}^{\text {shape }}=$ $E_{3 \times 7} / E_{7 \times 7}$ is calculated. The expression $E_{n \times m}$ stands for the energy deposited in a window of $n \times m$ around $\left(\eta_{1}, \phi_{1}\right)$. This shape variable exploits the fact that most of the energy of EM showers is deposited in the second sampling of the EM calorimeter.

- In the first sampling, the quantity $R_{\eta}^{\text {strip }=}$ $\left(E_{1 \mathrm{st}}-E_{2 \mathrm{nd}}\right) /\left(E_{1 \mathrm{st}}+E_{2 \mathrm{nd}}\right)$ is obtained in a window of $\Delta \eta \times \Delta \phi=0.125 \times 0.2$ around $\left(\eta_{c}, \phi_{c}\right) . E_{1 \mathrm{st}}$ and $E_{2 \mathrm{nd}}$ are the energies of the two highest local maxima found, obtained on a strip-by-strip basis. The two $\phi$-bins are summed and only the distribution in $\eta$ is considered. A local maximum is defined as a single strip with an energy greater than its two adjacent strips.

- The total transverse energy $E_{\mathrm{T}}$ deposited in the EM calorimeter is calculated in a window of $3 \times 7 \mathrm{Cell}$ s around $\left(\eta_{1}, \phi_{1}\right)$.

- Finally, the energy that leaks into the hadron calorimeter $E_{T}^{\text {had }}$ is calculated in a window of size $\Delta \eta \times \Delta \phi=0.2 \times$ 0.2 around $\left(\eta_{c}, \phi_{c}\right)$.

\section{F. Level-2 Muon Reconstruction: muFast}

The muFast [17] algorithm is a Level-2 track reconstruction algorithm for the Muon Spectrometer. The algorithm is seeded by the Level-1 muon RoI and uses both RPC and MDT measurements. At present, this algorithm is limited to the barrel region and functions in the following sequence:

- an emulation of the Level-1 muon trigger is performed to extract RPC hits that induced the Level-1 trigger accept decision;

- pattern recognition is performed using RPC hits to define a road in the MDT chambers around a putative muon trajectory. MDT tubes lying within the road are selected, and a contiguity algorithm is applied to remove background hits not associated with the muon trajectory;

- a straight-line track fit is made to the selected tubes (one per each tube monolayer) within each MDT station; for 
TABLE III

Performance of THE Single-Electron HLT at AN LHC BEAM LuMinosity of $2 \times 10^{33} \mathrm{CM}^{-2} \mathrm{~s}^{-1}$. THE RESUlTS ARE PRESENTED IN A Single SEQUence. MATCHING REFERS TO POSITION AND ENERGY/MOMENTUM MatCHING BeTWEen CALORIMETER Clusters and RECONSTRUCTED

Tracks (N.B. AT LEVEL-2 ONLY TRACKS Found With THE PIXEL AND SCT Detectors ARE Used). The EFFiciencies ARE Given For Single

ELECTRONS OF $p_{\mathrm{T}}=25 \mathrm{GeV} / \mathrm{c}$ ALREADY SELECTED By LEVEL-1, AVERAGED OVER THE FUll PSEUDORAPIDITY RANGE $\|\eta\|<2.5$. FOR REFERENCE, THE EFFICIENCY OF THE LEVEL-1 SELECTION IS 95\%. THE RATES ARE NORMALIZED TO A LEVEL-1 RATE FOR EM CLUSTERS OF ROUGHLY $7.7 \mathrm{kHz}$

\begin{tabular}{c|c|c}
\hline Trigger Step & Rate $(\mathrm{Hz})$ & Cumulative Efficiency (\%) \\
\hline Level-2 Calorimeter & $1.9 \times 10^{3}$ & 96 \\
Level-2 Tracking & $0.4 \times 10^{3}$ & 89 \\
Level-2 Matching & 140 & 88 \\
\hline EF Calorimeter & 100 & 86 \\
EF Tracking & 71 & 82 \\
EF Matching & 34 & 80 \\
\hline
\end{tabular}

this procedure, the drift-time measurements are used to exploit the high measurement accuracy of the muon tracking system; the track sagitta is then evaluated;

- a fast $p_{\mathrm{T}}$ estimate is made using a LUT; the LUT encodes the linear relationship between the measured sagitta and the $q / p_{\mathrm{T}}$ (where $q$ denotes the charge of the muon), as a function of $\eta$ and $\phi$.

\section{G. Event Filter Algorithms}

Event filter algorithms consist of algorithms imported directly from those developed in the offline software; they are described in detail elsewhere [3], [18]. Two complementary InDet track reconstruction packages are used: xKalman++ [13] and iPatRec [19]. The package egammaRec is designed to combine tracking information with information from Caloclusters found by the two dedicated algorithms for reconstructing CaloClusters in the LAr and Tile calorimeters: the LArClusterRec and TileRec packages, respectively. A track reconstruction package for the Muon Spectrometer, Moore(Muon Object Oriented REconstruction) uses collections of digits or clusters from the MDT chambers in the Muon Spectrometer to find and fit tracks [20].

\section{Performance Studies}

This section presents an assessment of the physics performance of the trigger algorithms for Level-2 and EF described in Section III and applied to two representative classes of finalstates: electrons and photons; and muons. Relevant aspects of system performance are also mentioned in each of the two following subsections.

\section{A. Electrons and Photons}

The inclusive electron and photon triggers are expected to contribute a substantial fraction of the total high- $p_{\mathrm{T}}$ trigger rate. After the selection by Level-2 and the EF, the remaining rate will contain a significant contribution from Standard Model physics processes containing real isolated electrons or photons.

At Level-1, electrons and photons are selected using calorimeter information with reduced granularity. After receiving the Level-1 electromagnetic (EM) trigger RoI positions,

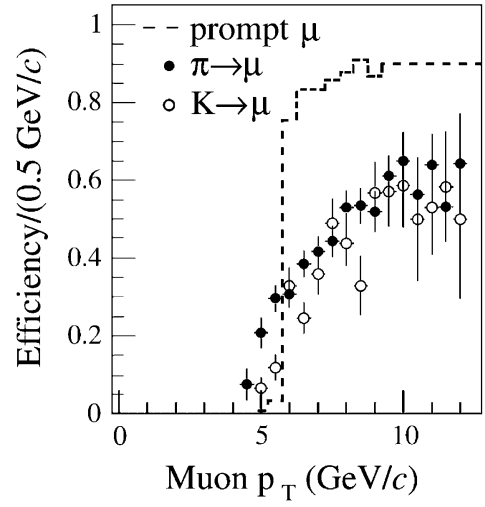

Fig. 3. The Level-2 muon reconstruction efficiency (with respect to Level-1) using the standalone algorithm muFast combined with Inner Detector tracks at an LHC luminosity of $1 \times 10^{33} \mathrm{~cm}^{-2} \mathrm{~s}^{-1}$. A lower $p_{\mathrm{T}}$ threshold of $6 \mathrm{GeV} / c$ is required for muon candidates at Level-2.

the Level-2 trigger performs a selection of isolated EM clusters using the full calorimeter granularity within an RoI of size $\Delta \eta \times \Delta \phi=0.2 \times 0.2$. As described in Section III-E, electrons and photons are selected based on EM cluster $E_{\mathrm{T}}$ and shower-shape quantities that distinguish isolated EM objects from jets. A further, more refined calorimeter-based selection may classify the EM cluster as a candidate photon trigger object. In the next step, electrons are identified by associating the EM cluster with a track in the InDet. In general, track candidates are found by independent searches in the TRT and SCT/Pixel detectors in the region identified by the Level-1 RoI. For the results presented in the next section, however, only IDSCAN (Section III-A), has been used; work is in progress to evaluate the other algorithms. For electron candidates, a matching of position and momentum measurements between the track and the cluster is required. Electron candidates passing the Level-2 selection criteria are retained to be examined by the EF.

In the EF, electrons are selected with a strategy similar to that for Level-2 using information from the calorimeters and the InDet. For track reconstruction, results obtained with xKalman++ (Section III-G) are presented below; work is in progress to evaluate the alternative track reconstruction program iPatRec (Section III-G). First results show similar behavior between these two algorithms. The main differences with respect to Level-2 arise from the availability of calibration data and the possibility to use more sophisticated reconstruction algorithms with access to the detector data for the full event. This results in sharper thresholds and better background rejection. In order to avoid biases introduced by using different reconstruction algorithms for online and offline selection, the EF will select events using the offline reconstruction algorithms to the largest possible extent. However, using the offline reconstruction in the EF implies that the offline algorithms must comply with the stricter EF requirements in terms of robustness and system performance.

The performance of the electron and photon triggers has been estimated for single electrons and photons, and for some standard physics channels (e.g., $\mathrm{Z} \rightarrow \mathrm{e}^{+} \mathrm{e}^{-}, \mathrm{W} \rightarrow \mathrm{e} \nu, \mathrm{H} \rightarrow \mathrm{ZZ} \rightarrow$ $\mathrm{e}^{+} \mathrm{e}^{-} \mathrm{e}^{+} \mathrm{e}^{-}$). The physics performance is characterized in terms of efficiency for the signal channel, and the rate expected for the 
selection. Currently, only single electron triggers are studied. Rate and efficiencies are presented for each step of the Trigger process in Table III. Further details of similar studies can be found in [3].

Studies of the system performance of algorithms and associated components of the HLTSSW described in Section II are currently underway. Preliminary studies have been conducted, and, for Level-2 algorithms, may be disaggregated along the sequence presented in Fig. 2. In each case, the computation time needed is expressed in terms of execution speed on a personal computer with a CPU speed of between 1 and 2 GHz. The Region Selector (Section II-E) requires less than $1 \mathrm{~ms} / \mathrm{RoI} / \mathrm{subdetector}$. The access to data via the ROB Data Collector requires on the order of $100 \mu \mathrm{s}$. Within an RoI low luminosity, the conversion of raw data ByteStream into EDOs currently requires roughly 5,4 , and $6 \mathrm{~ms}$ for the SCT/Pixel, LAr, and Muon Spectrometer subdetectors, respectively. To process data within Level-2 algorithms for the electron and photon selection is at maximum on the order of a few milliseconds. Hence, all components perform adequately within the $10 \mathrm{~ms}$ average Level-2 latency extrapolated to an $8-\mathrm{GHz}$ CPU with the exception of the raw data conversion. Work is currently in progress to reduce this time to an acceptable level; preliminary results are encouraging.

\section{B. Muons}

The muon trigger selection strives to accurately reconstruct muon tracks within RoIs indicated by the Level-1 muon trigger. Level-2 and the EF must reject low- $p_{\mathrm{T}}$ muons (i.e., muons with $p_{\mathrm{T}}$ below the threshold that are initially selected due to the Level-1 limited resolution), secondary muons produced by decays-in-flight of charged pions and kaons, and fake muon tracks composed of hits from the cavern background.

The tracks found in the Level-2 muon trigger are extrapolated and combined with measurements from the InDet and the calorimeters. Matching between muon tracks measured independently in the muon system and the InDet selects prompt muons and rejects fake and secondary muons. Using the Level-2 muFast algorithm (Section III-F), a muon $p_{\mathrm{T}}$ resolution of $4 \%$ is obtained. Fig. 3 shows the efficiency reconstruction as a function of $p_{\mathrm{T}}$ for prompt muons as well as secondary muons from $\pi^{ \pm}$and $K^{ \pm}$decays-in-flight.

\section{SUMmARY AND CONCLUSION}

The future LHC at CERN offers unprecedented challenges to the design and construction of detectors and trigger/DAQ systems. For ATLAS, a three-level trigger system has been developed to extract interesting physics signatures with a $10^{6}$ rate reduction. To accomplish this, components of physics analysis traditionally deferred to offline physics analysis will be embedded within the online trigger system.

A dedicated and specialized selection software framework has been designed for the HLT. Algorithms will operate within this framework and must cope with data access and latency limitations. Further development and tests of this model along with a broad suite of algorithms using a realistic raw data access paradigm are underway.

\section{ACKNOWLEDGMENT}

We wish to acknowledge and thank the ATLAS Offline Software community for their many contributions to the high-level trigger selection software framework, as well as high-level trigger algorithms. Crucial efforts to the establishment of a realistic raw data access paradigm for the various subdetectors were made by M. Cobal, H. Ma, L. Santi, J. Schieck, A. Solodkov, A. Zalite, and Y. Zalite. The realization of the region selection mechanism was made in collaboration with $\mathrm{K}$. Assamagan, G. Gorfine, F. Luehring, H. Ma, and A. Solodkov.

\section{REFERENCES}

[1]. The ATLAS High-Level Trigger Group. [Online]. Available: http:// atlas.web.cern.ch/Atlas/GROUPS/DAQTRIG/HLT/AUTHORLISTS/ rt2003.pdf

[2] "ATLAS Detector and Physics Performance Tech. Design Rep.," ATLAS Collaboration, CERN/LHCC/99-14, 1999.

[3] "ATLAS High-Level Trigger, Data Acquisition and Controls: Tech. Design Rep.," ATLAS HLT/DAQ/DCS Groups, ATLAS TDR-016, 2003.

[4] S. Armstrong et al., "Requirements for an Inner Detector Event Data Model,", ATLAS Internal Note ATL-DAQ-2002-011, 2002.

[5] Athena: The ATLAS Common Framework User Guide and Tutorial [Online]. Available: http://atlas.web.cern.ch/Atlas/GROUPS/SOFTWARE/OO/Development/

[6] S. George, T. Hansl-Kozanecka, K. Mahboubi, and A. Watson, "RoI-Based Event Descriptions for Modeling the ATLAS Second Level Trigger,", ATLAS Internal Note ATL-COM-DAQ-99-011.

[7] S. Armstrong et al., "A new implementation of the region-of-interest strategy for the ATLAS second level trigger," in Proc. 2003 Conf. Computing in High Energy and Nuclear Phys., La Jolla, CA, 2003.

[8] A. Schaffer and C. Arnault, "Use of a generic identification scheme connecting events and detector description in ATLAS," in Proc. 2003 Conf. Computing in High Energy and Nuclear Physics, La Jolla, CA, 2003.

[9] G. Commune et al., "The algorithm steering and trigger decision mechanism of the ATLAS high level trigger," in Proc. 2003 Conf. Computing in High Energy and Nuclear Phys., La Jolla, CA, 2003.

[10] H. Drevermann and N. Konstantinidis, "Determination of the z position of primary interactions in ATLAS,", ATLAS Internal Note ATL-DAQ2002-014, 2002.

[11] _ - "Algorithms to select space points of tracks from single primary interactions in ATLAS,", ATLAS Internal Note ATL-COM-DAQ2003-040, 2003.

[12] M. Cervetto, P. Morettini, F. Parodi, and C. Schiavi, "iTrack: A Level-2 track reconstruction algorithm based on silicon detectors,", ATLAS Internal Note ATL-COM-DAQ-2003-025, 2003.

[13] I. Gavrilenko, "Description of global pattern recognition program (xKalman),", ATLAS Internal Note ATLAS-INDET-97-165, 1997.

[14] J. Baines et al., "Global pattern recognition in the TRT for B-physics in the ATLAS trigger,", ATLAS Internal Note ATL-DAQ-99-012, 1999. M. Sessler, M. Smizanska, "Global pattern recognition in the TRT for the ATLAS Level-2 trigger," ATLAS Internal Note ATL-DAQ-98-120.

[15] S. González, T. Hansl-Kozanecka, and M. Wielers, "Selection of high- ${ }_{T}$ Electromagnetic Clusters by the Level-2 Trigger of ATLAS,", ATLAS Internal Note ATL-DAQ-2000-002, 2000. S. González, B. González Pineiro, and T. Shears, "First implementation of calorimeter FEX algorithms in the Level-2 reference software," ATLAS Internal Note ATL-DAQ-2000-020; S. González, T. Shears, "Further studies and optimization of the level-2 trigger electron/photon FEX algorithm," ATLAS Internal Note ATL-DAQ-2000-042.

[16] ATLAS Calorimeter Perform., 1996.

[17] A. di Mattia et al., "A Muon Trigger Algorithm for Level-2 Feature Extraction,", ATLAS Internal Note ATL-DAQ-2000-036, 2000. A. di Mattia, S. Falciano, A. Nisati, "The implementation of the $\mu$ fast algorithm in the new PESA framework," ATLAS Internal Note ATL-DAQ2003-021.

[18] S. Armstrong et al., "Algorithms for the ATLAS High-Level Trigger,", ATLAS Internal Document ATL-DAQ-2003-002, 2003.

[19] iPatRec: Inner detector pattern recognition and track fitting, R. Clifft and A. Poppleton. [Online]. Available: http://atlasinfo.cern.ch/ Atlas/GROUPS/SOFTWARE/DOCUMENTS/IPATREC/ipatrec.html

[20] Moore-muon OO REconstruction for ATLAS [Online]. Available: http://www.usatlas.bnl.gov/computing/software/moore/ 